\title{
Changement Climatique Et Paludisme En Côte d'Ivoire : Représentations Sociales Et Connaissance Des Populations d'Adjéyaokro (Bouaké)
}

\author{
Mazou Gnazegbo Hilaire \\ Université Alassane Ouattara \\ Département d'Anthropologie et Sociologie, Côte d'Ivoire
}

doi: 10.19044/esj.2017.v13n26p110 URL:http://dx.doi.org/10.19044/esj.2017.v13n26p110

\begin{abstract}
Climate change characterized by increasing average daily temperature and changing seasons has become a public health concern, with the incidence of malaria in recent years. Despite research on global warming and efforts to fight malaria (sensitization campaigns, distribution of insecticide-treated nets, care of the sick), the excessive heat continues to do damage. What is the link between climate change and malaria? What are people's perceptions of climate change in relation to malaria?

To address these concerns, the survey on the impact of climate change on malaria was conducted through in-depth interviews with some 20 people from the village of Adjéyaokro in Bouaké. It is mainly the result of our investigations that the populations do not link the effects of climate change and the risk of exposure to malaria.
\end{abstract}

Keywords: Climate change, global warming, malaria, living environment, risks of exposure to malaria, human behavior

\section{Résumé}

Le changement climatique caractérisé par l'augmentation de la température moyenne journalière et le changement des saisons, est devenu un sujet de préoccupation de santé publique, avec l'incidence du paludisme au cours de ces dernières années. En dépit des recherches sur le réchauffement planétaire et les efforts pour lutter contre le paludisme (campagnes de sensibilisations, distribution de moustiquaires imprégnées, prise en charge des malades), la trop forte chaleur continue de faire des dégâts. Quel est donc le lien entre changement climatique et paludisme ? Quelle est la perception que les populations ont du changement climatique en rapport avec le paludisme? 
Pour répondre à ces préoccupations, l'enquête sur l'incidence du changement climatique sur le paludisme a été conduite au travers d'entretiens approfondis, auprès d'une vingtaine de personnes du village d'Adjéyaokro à Bouaké. Il ressort principalement de nos investigations que les populations ne font aucun lien entre les effets du changement climatique et les risques d'expositions au paludisme.

Mots clés : Changement climatique, réchauffement climatique, paludisme, cadre de vie, risques d'exposition au paludisme, comportements humains

\section{Introduction}

Le changement climatique représente désormais le défi le plus important auquel se trouve confrontée toute la planète terre. Inondations, sécheresses, fontes de glaciers sont caractéristiques de ces changements climatiques globaux. Également, la chute des précipitations ainsi que la hausse des températures terrestres avec leurs corollaires (réduction de la production agricole, détérioration de la sécurité alimentaire, incidence accrue des inondations et de la sécheresse, propagation des maladies et augmentation du risque de conflits en raison de la saturation foncière) en constituent les éléments les plus ressentis par les populations rurales africaines (Trape, 2005 ; OMS ${ }^{1}, 1996$; Youphil, 2014 ; Valette, 2015).

Les changements climatiques seraient la cause d'un éventail de catastrophes environnementales et sanitaires. Cette idée semble vérifiée dans le cas de plusieurs maladies dont le paludisme qui est endémique dans les régions chaudes. En effet, le paludisme constitue l'une des premières causes de morbidité par maladie vectorielle dans une grande partie de l'Afrique subsaharienne. Elle constitue aussi l'une des maladies tropicales dont l'épidémiologie a été la plus affectée ces dernières années par le changement climatique (Patz et al., 2006; Minakawa et al., 2002; OMS, 2015). Le système sanitaire de la Côte d'Ivoire, pays situé dans la zone tropicale, est aussi confronté à une recrudescence de grandes maladies tropicales (PNLP, 2008 ; OMS, 2009). De toutes ces épidémies, le paludisme représente un problème majeur de santé publique avec l'augmentation des températures et des précipitations en lien avec le changement climatique (Capelle, 2007).

Les populations de de Bouaké ${ }^{2}$, n'échappe pas à ce triste et morose tableau. En effet, cette zone connaît une épidémiologie fortement typée par sa géographie avec une forte dominante de maladies infectieuses et

${ }^{1}$ Organisation Mondiale de la Santé

${ }^{2}$ Deuxième grande ville située au centre de la Côte d'Ivoire, chef-lieu de la région de Gbêkê 
parasitaires qui représentent $50 \%$ à $60 \%$ de la morbidité observée (PNDS ${ }^{3}$, 1996 : 8 ; PNLP ${ }^{4}, 2010$; OMS, 2010).

Dans cette localité, les changements importants dans les conditions climatiques locales comprennent des conditions chaudes et davantage ensoleillées en période de saison sèche. Aussi, pour les populations de Bouaké, la chaleur, participent à la récurrence de nombreux cas de fièvre qui débouchent inévitablement sur des états palustres (PNLP, 2010). Ainsi, la compréhension du lien entre le changement climatique et l'incidence du paludisme, chez les populations locales, se pose comme problème. Comment ces populations se représentent-elles le paludisme et le changement climatique ? Quelles sont les caractéristiques du changement climatique chez les populations ? Quel est le lien entre le changement climatique et l'incidence du paludisme?

Pour répondre à toutes ces préoccupations, les données relatives au changement climatique en lien avec le paludisme, ont été collectées à partir d'une enquête qualitative menée auprès de la communauté villageoise d'Adjéyaokro.

\section{Méthodologie de la recherche}

Cette recherche, se propose d'analyser l'impact du changement climatique sur l'incidence du paludisme chez les populations de Bouaké. L'enquête de terrain, s'est déroulée dans le village d'Adjéyaokro situé à l'ouest de la ville de Bouaké, sur l'axe Bouaké-Béoumi, non loin du de l'université Alassane Ouattara. Le choix du village d'Adjéyaokro pour cette recherche, est non seulement lié à sa situation géographique, mais aussi à sa forte concentration démographique en termes de population d'étudiants. L'enquête s'est intéressée à toutes les catégories socioprofessionnelles que comptent le village d'Adjéyaokro. Il s'agit notamment, des paysans, des hommes de métier, des fonctionnaires, des ménagères et des étudiants. Le travail de terrain s'est déroulé entre le 13 juillet et le 21 Aout 2015. D'abord, l'enquête exploratoire a permis de cibler les personnes au profil pertinent (vieux, jeunes, hommes et femmes) pour comprendre en profondeur le rapport entre changement climatique et paludisme, en vue de faciliter l'enquête de terrain. C'est sur cette base que nous avons eu, avec les 20 enquêtés, des entretiens directs et souvent informels, structurés autour de certaines thématiques telles que le niveau de connaissance sur le changement climatique, le paludisme et le lien entre changement climatique et paludisme. Ainsi, nous avons pu réunir un ensemble de témoignages qui permet

\footnotetext{
${ }^{3}$ PNDS : Programme National de Développement Sanitaire

${ }^{4}$ Programme National de Lutte contre le Paludisme
} 
d'apprécier les représentations sociales autour du paludisme et du changement climatique en lien avec l'incidence du paludisme.

La problématique du changement climatique a fait l'objet de plusieurs travaux, tant sur son impact sanitaire, les considérations d'ordre scientifique, social et environnemental, que sur les mesures d'adaptation des populations face à ce phénomènes (Patz et al, 2006 ; OMS, 2015). D’abord, plusieurs études indiquent qu'il existe trois types d'effets sanitaires indirectement liés au changement climatique. Il s'agit notamment des maladies à transmission vectorielle (la maladie du Nil occidental, paludisme, dengue...), les maladies liées à la raréfaction et à la pollution de l'eau (fièvre typhoïde, choléra, salmonelloses...) et les maladies liées à la pollution de l'air tel que l'asthme (Besancenot, 2007). Particulièrement, les moustiques sont très sensibles aux changements du climat. Les conditions plus chaudes permettent aux moustiques et au parasite du paludisme de se développer plus rapidement, alors que les conditions plus humides leurs permettent de vivre plus longtemps et de se reproduire de manière plus importante (Mahande et al, 2007).

Ensuite, Presque tous les rapports des grandes conférences sur le changement climatique, ont abordé pratiquement le volet ''adaptation et atténuation'. Les mesures donc d'adaptation sont multiples. Certaines ont été déjà appliquées pendant que d'autres sont en projet (AFR/RC64/INF.DOC/4, 2014 ; AFR/RC61/10. 2011).

Tous les travaux cités plus haut, ont largement traité de l'impact du changement climatique ainsi que des mesures d'adaptation de la santé publique. Cependant, il ressort que les représentations des populations directement concernées, ne soient pas suffisamment prises en compte, alors que le cadre de vie et les comportements humains augmentent les risques d'expositions au paludisme en lien avec le changement climatique.

Les informations collectées ont par la suite, été analysées selon l'éclairage de la théorie de la représentation sociale qui se présente comme un ensemble d'informations, de croyances, d'opinions et d'attitudes propres à un groupe donné, à propos d'un objet donné. Les représentations sociales remplissent à la fois une fonction de savoir, une fonction identitaire et une fonction d'orientation. C'est une forme de connaissance socialement élaborée et partagée, ayant une visée pratique et encourageant à la construction d'une réalité commune à un ensemble social (Jodelet, 1989). Cette théorie a permis de comprendre et d'analyser la signification sociale du paludisme en lien avec le changement climatique et les contextes socioculturels et historiques qui influencent les idées et les actions quotidiennes des populations d'Adjéyaokro. 


\section{Résultats de la recherche}

\section{Le paludisme, une maladie aux contours non encore maitrisés par les populations}

L'enquête de terrain a montré que le paludisme représente un réel problème de santé pour les populations d'Adjéyaokro. Malgré la gravité et la persistance de cette maladie, la plupart des enquêtés pensent, à travers leurs différents propos, que le soleil et l'alimentation seraient à la base de l'incidence du paludisme à Adjéyaokro.

« Soleil de maintenant là, il pique jusqu'à il touche os qui est dans corps de l'homme-là, nous on est toujours au soleil, on travaille en bas, nos enfants marchent sur soleil là pour aller à l'école ; c'est dans ça ils attrapent palu. Donc c'est soleil qui donne palu à les gens » (MC, ferronnier),

"Il y a des mangés aussi qui provoquent palu hein, il faut qu'on se dise la vérité, Ya des huiles qui sont au marché là ; des fois quand tu vois huile là dans bouteille, si tu ne secoues pas dans peu de temps-là ça devient on dirait beurre de karité. Bon, quelqu'un tu consommes ça et tu sors en bas de soleil, quand soleil là va chauffer c'est comme si tu as mis huile - là au feu dans ton corps là-bas. Même quand tu manges attiéké, huile que je parle là faut regarder en bas dans assiette là, ça va rester coller. Au nom de Dieu ça peut provoquer palu, ça c'est une réalité »(SA, paysan)

Dans le même ordre d'idée, " nous nos parents disent que temps de maïs là, quand tu manges beaucoup de maïs frais, ça te donne palu et puis huile rouge aussi. Remarque bien palu jaune là c'est ceux qui mangent beaucoup huile rouge-là qui ont ça » (Dame K, ménagère).

Selon les enquêtés, la consommation de l'huile de mauvaise qualité, du maïs frais, de l'attiéké, de l'huile rouge et des mangues vertes, constitue la principale cause du paludisme. En effet, les enquêtés soutiennent que le maïs frais et les mangues vertes, consommés abondamment, provoqueraient des remontés gastriques et des vomissements de couleur jaune symptôme du paludisme. L'huile rouge quant à elle, serait à la base du jaunissement des yeux, des paumes et des urines. L'attiéké qui représente la nourriture de base des populations d'Adjéyaokro, est vu comme source de constipation donc de 
paludisme. Telle est la signification sociale du paludisme chez les populations d'Adjéyaokro. Qu'en est-il du changement climatique ?

\section{Le changement climatique, un phénomène méconnu des populations}

La recherche a montré que le changement climatique apparait comme un phénomène nouveau, donc méconnu des populations d'Adjéyaokro. Les représentations sociales liées au changement climatique sont justement traduites par les différents propos des enquêtés.

"Le changement climatique c'est l'élévation de la température, très souvent, au moment où on s'attend à la pluie, c'est la chaleur totale et quand on pense entrer dans les périodes de chaleur, c'est la pluie qui se manifeste »(TH, Fonctionnaire).

"Vrai vrai là, faut pas qu'on va se mentir cadeau, je n'ai aucune notion $d u$ changement climatique je ne blague pas les amis » (Mlle C, Etudiante).

Pour les enquêtés d'Adjéyaokro, les causes du changement climatique sont multiples. Il s'agit notamment des différents comportements des hommes dans la vie de tous les jours. Comme l'attestent ces différents propos.

"Le changement climatique est provoqué par nous-mêmes. Les fumées des usines, les gaz d'échappement des voitures, les produits chimiques, ce sont les effets néfastes de ces produits qui provoquent le changement climatique » (MK, Enseignant).

"C'est parce qu'ils ont trop fait du mal à Bouaké ici qu'il ne pleut pas. Ils ont trop versé du sang ici oh; donc les dieux sont fâchés »(AH, Etudiante).

"Les gens ont trop fait couler le sang dêh! Ils font ce qui n'est pas bien et on ne faisait pas les mauvaises choses avant. Donc s'il ne pleut pas ou si le temps est comme ça, c'est à cause de ça. Donc ça là, c'est Dieu seul qui peut nous sauver » (MC, Commerçant).

Pour ces populations le changement climatique est fortement lié au comportement humain qui concerne notamment, les agissements de l'homme 
sur l'environnement et surtout les atrocités et crimes commis pendant les différentes crises que la Côte d'Ivoire a connu ces dernières années.

\section{Aucun lien entre changement climatique et paludisme chez les populations d'Adjéyaokro.}

Les informations collectées au cours de cette recherche, ont permis de montrer que les populations d'Adjéyaokro n'établissent aucun lien entre le changement climatique et les risques d'exposition au paludisme. En effet pour ces enquêtés, il n'y a aucun rapport entre le changement climatique et le paludisme dans la mesure où le paludisme est causé par le soleil, l'alimentation et la fatigue liée aux activités humaines. Ainsi selon ces enquêtés, il est impossible que le paludisme ait, un quelconque lien d'avec le changement climatique qu'ils considèrent dans la plupart des cas, comme étant l'expression de la colère divine. Cette manière de penser le lien entre changement climatique et paludisme est traduite par leurs différents propos.

Ainsi pour cet enquêté, la chaleur n'a aucun lien avec le paludisme, "Pour moi hein, il n'y a pas de lien parce que vous pouvez être dans une maison, je prends un exemple terre à terre (...), chez vous par exemple, il y a des gens qui préfèrent les moments de chaleur. C'est-àdire quand il fait chaud, ils se sentent à l'aise. Par contre, il y a d'autres qui préfèrent les moments de fraîcheur, quand il fait froid, il est plus à l'aise parce qu'il transpire moins. Maintenant il faut voir un peu. Si la chaleur provoquait le paludisme tous ceux qui préfèrent les périodes de chaleur là ils seraient tous à l'hôpital» (MK, Enseignant).

Selon Melle F, le paludisme serait uniquement lié à la consommation excessive de l'huile.

"Le palu vient quand on mange beaucoup

Dans la même logique, d'huile » (Melle F, non scolarisée).

"Le paludisme est dù à la nourriture et à la fatigue. Soleil donne palu aussi " (O, élève en classe de $3^{\text {ème}}$ )

L'enquête de terrain a ainsi montré que les enquêtés ne font aucun lien entre le changement climatique et les risques d'exposition au paludisme. Cela est dû au fait qu'ils ignorent non seulement, les causes du paludisme mais aussi et surtout, les effets du changement climatique. 


\section{Analyse et discussion}

Plusieurs écrits ont montré que le paludisme est une maladie transmise par les piqûres de moustiques. Il est aussi la première des endémies pour lesquelles l'humanité paye un lourd tribut. En effet, chaque année, environ 300 à 500 millions de nouveaux cas sont enregistrés, 1 à 3 millions de personnes en meurent, surtout en Afrique subsaharienne (OMS, 2009; OMS, 2015). En Côte d'Ivoire, le paludisme constitue le premier motif de consultation et l'une des premières causes de mortalité ${ }^{5}$ et de morbidité ${ }^{6}$, surtout chez les femmes enceintes et les enfants de moins de cinq ans. Il constitue la cause principale d'absentéisme scolaire et professionnel, de réduction de la productivité et des revenus (Mazou, 2008; PNLP, 2008). Le paludisme tue plus de gens aujourd'hui qu'au cours des trois dernières décennies (Mouchet et al., 1998 ; Capelle, 2007 ; OMS. 2009).

Malgré cette documentation sur le paludisme, la plupart des enquêtés, pensent encore que l'alimentation, le soleil et la fatigue constituent les principales causes du paludisme. Pour notre part, le niveau de connaissance des populations d'Adjéyaokro vis-à-vis des risques d'exposition au paludisme est fortement lié à leurs différentes représentations. Ces populations ignorent donc dans l'ensemble, que les piqûres de moustiques sont responsables de la transmission du paludisme.

A partir de ce constat, notre recherche se présente sous l'angle de la représentation sociale et connaissance du paludisme en lien avec le changement climatique. Dans le contexte particulier d'Adjéyaokro, la transmission ou l'émergence du paludisme, n'est pas liée aux piqûres de moustiques, mais plutôt à certains déterminants comme le soleil, la fatigue et l'alimentation (Mazou, 2008; OMS, 2010). La saison sèche réunit les conditions bio-écologiques propices au développement des moustiques (anophèles) vecteurs du paludisme, c'est également la période où le soleil apparait de façon ardente (Darrief, 1998 ; Charmot, 1982 ; Carneval, 1998). La saison sèche est aussi une période d'intense activité agricole source de fatigue. Or, la fatigue entraîne une baisse de la résistance de l'organisme aux maladies, notamment le paludisme (Nzeyimana et al, 2002 ; Charmot, 1982 ; Mouchet et Carnevale, 1998). Pourtant, les populations d'Adjéyaokro en établissant le lien entre le soleil, l'effort physique et le paludisme, ne tiennent pas compte des moustiques qui sont en réalité responsables de la maladie. En effet, à partir d'une piqûre de moustique, le paludisme présent de façon latente dans l'organisme se développe sous l'influence du soleil, de l'activité physique, de l'humidité... (Mouchet et al., 2004).

\footnotetext{
${ }^{5}$ Phénomène de la mort, considéré du point de vue du nombre. C'est aussi le rapport des décès dans une population à l'effectif moyen dans celle-ci durant une période donnée.

${ }^{6}$ Rapport entre le nombre des malades et celui d'une population.
} 
De plus, les populations d'Adjéyaokro, ont d'abord une méconnaissance du changement climatique. Ensuite, elles n'établissent aucun lien entre le changement climatique et les risques d'exposition au paludisme contrairement à la littérature sur la question. Dans la plupart des cas, ces populations considèrent les effets du changement climatique comme étant l'expression de la colère divine, une punition.

En revanche, les activités humaines telles que les transports, l'industrie, la production d'énergie, l'agriculture ou encore le chauffage, libèrent de nombreux gaz. Certains, comme le dioxyde de carbone (CO2), le méthane $(\mathrm{CH} 4)$ ou le protoxyde d'azote $(\mathrm{N} 2 \mathrm{O})$, piègent dans l'atmosphère une partie de la chaleur émise par la planète sous forme de rayons infrarouges. Ils sont appelés gaz à effet de serre (GES). Cet effet physique naturel permet à la terre d'avoir une température moyenne globale de $15^{\circ} \mathrm{C}$ au lieu de $-18^{\circ} \mathrm{C}$, permettant ainsi à la vie de se développer. Cependant, l'augmentation continue de la concentration de ces gaz depuis la révolution industrielle au milieu du 19e siècle renforce cet effet de serre et la température globale de la planète augmente. Ce réchauffement de l'atmosphère et des océans a un impact sur le climat, notamment sur la pluviométrie et les mouvements des masses d'air et des courants marins d'où l'utilisation du terme de « changement climatique » (Mouchet et al., 2004).

Le changement climatique désigne la variation des caractéristiques climatiques en un endroit donné et au cours du temps tels que le réchauffement ou le refroidissement. Ce changement climatique peut entraîner des dommages importants : élévation du niveau des mers, accentuation des événements climatiques extrêmes (sécheresses, inondations, cyclones, etc.), déstabilisation des forêts, menaces sur les ressources d'eau douce, difficultés agricoles, désertification, réduction de la biodiversité, extension des maladies tropicales, etc. (Dictionnaire, 2009).

Le changement climatique ne menace pas seulement la biodiversité mais aussi notre santé, avec des effets qui se font d'ores et déjà sentir et pourraient devenir catastrophiques si rien n'est fait pour limiter l'augmentation des températures. L'OMS prévoit ainsi, dès 2030, près de 250000 décès supplémentaires par an dus directement ou indirectement au réchauffement global, avec pour principales causes : la malnutrition, le paludisme, la diarrhée et le stress thermique (Watts et al., 2015; Rey et al., 2007). D'après les données actuelles, la variabilité du climat pourrait avoir une influence directe sur l'épidémiologie des maladies à transmission vectorielle dont le paludisme. En effet, le paludisme est l'une des maladies les plus importantes à transmission vectorielle dans les régions tropicales et subtropicales. Le changement climatique est déjà responsable d'environ $6 \%$ des cas de paludisme dans le monde et dans certains pays à revenu intermédiaire (OMS, 2010). 
La question du changement climatique en lien avec le paludisme est bien documentée. Mais malgré tout, les enquêtés ne font aucun lien entre ce phénomène et les risques d'exposition au paludisme. Dans un premier temps, cela s'explique par le fait que les populations enquêtées, méconnaissent non seulement les causes du paludisme, mais aussi et surtout, les effets du changement climatique. Ensuite, le faible niveau de connaissance des populations enquêtées sur le changement climatique en lien avec le paludisme est fortement lié à leurs différentes représentations sociales. En effet, dans le contexte particulier d'Adjéyaokro, les populations ont leurs propres informations, croyances, opinions et attitudes, à propos du paludisme et du changement climatique. Cette forme de connaissance socialement élaborée et partagée, permet de comprendre que les populations enquêtées, n'ont pas encore pris conscience que ces changements représentent un danger pour les activités économiques, les infrastructures et les écosystèmes aménagés. Elles ne reconnaissent pas encore les risques réels que représentent ces changements climatiques pour leur santé (Jodelet, 1989; Najera, 1989). En rapport avec le paludisme, notons que les moustiques sont particulièrement sensibles aux différentes variations du climat. En effet, la chaleur permet aux moustiques de se développer plus rapidement, alors que l'humidité leur permet de vivre plus longtemps et de se reproduire de manière plus importante (Mahande et al, 2007).

Ainsi, le niveau de connaissance et les représentations sociales des populations, qui constituent réel obstacle à la lutte contre le paludisme en lien avec les effets du changement climatique, méritent une place de choix dans la communication pour le changement de comportement.

\section{Conclusion}

Longtemps ignoré, le changement climatique est à la base de plusieurs catastrophes environnementales et sanitaires qui s'accumulent de manière alarmante. Il s'agit notamment, des sécheresses, des inondations, de la déstabilisation des forêts, des difficultés agricoles, de la réduction de la biodiversité et de l'extension des maladies tropicales dont le paludisme. Le paludisme constitue l'une des maladies tropicales dont l'épidémiologie a été le plus affectée ces dernières années par le changement climatique. Cependant, il ressort de cette investigation, que les populations d'Adjéyaokro ne font aucun lien entre les effets du changement climatique et le paludisme alors que le cadre de vie et les comportements humains augmentent les risques d'expositions au paludisme en lien avec le changement climatique. Le faible niveau de connaissance des populations enquêtées sur le changement climatique et le paludisme est fortement lié à leurs différentes représentations sociales, leurs croyances en lien avec le manque d'informations. 
Des études ultérieures permettront sans nul doute, d'influencer qualitativement les représentations sociales, les connaissances et les attitudes des populations vis-à-vis du changement climatique et du paludisme. Pour cela, il faudra mettre l'accent sur la communication pour le changement de comportement, la mobilisation communautaire et la collaboration intersectorielle. En vue de fournir des informations sur l'importance des facteurs climatiques dans la santé publique et contribuer ainsi, à l'orientation des stratégies de lutte contre les effets du changement climatique et le paludisme.

\section{References:}

1. AFR/RC61/10, 2011. Cadre pour adaptation de la santé publique au changement climatique en africaine. Abidjan

2. AFR/RC64/INF.DOC/4, 2014. Programme panafricain pour l'adaptation de la santé publique au changement climatique: situation actuelle et perspectives. Cotonou

3. Besancenot J.-P., 2007. Changement climatique et impacts sanitaires : Une évolution déjà observable, Air pure ${ }^{\circ} 72,8 p$.

4. Capelle A. 2007. "Paludisme et réchauffement climatique», Mémoire de $2^{\text {ème }}$ année, certificat international d'écologie humaine, option écologie humaine et santé, université Paul Cézanne AixMarseille.

5. Charmot G.,1982. Paludisme: définir le type pour adapter le traitement, Afrique Santé, n¹6, pp. 21-26.

6. Darrief F., 1998. La lutte contre les moustiques nuisant et vecteurs de maladies, Paris, Karthala-ORSTOM

7. Dictionnaire, 2009. Dictionnaire environnement et développement durable, www.dictionnaire -environnement.com.

8. Jodelet D., 1989. Folies et représentations sociales. Paris, PUF

9. Mahande, A. et al., 2007. Feeding and resting behaviour of malaria vector, Anopheles arabiensis with reference to zooprophylaxis, Malaria Journal, 6, p. 100.

10. Mazou H., 2008. Approche socio-anthropologie du recours à la moustiquaire imprégnée dans la lutte contre le paludisme en Côte d'Ivoire: Cas de la zone forestière de Taï, Thèse de Doctorat Nouveau Régime de l'Université de Bouaké (Côte d'Ivoire), 307 p.

11. Minakawa N., et al., 2002. The effects of climatic factors on the distribution and abundance of malaria vectors in Kenya. J. Med. Entomol ;39 (6):833-841.

12. Mouchet J. et Carnevale P., 1998. Le paludisme, composante de l'environnement africain, ORSTOM actualités, $\mathrm{n}^{\circ} 20,8 \mathrm{p}$. 
13. Mouchet J., Carnevale P., Coosemans M. et al., 2004. Climat et paludisme. In : Biodiversité du paludisme dans le monde. John Libbey, Paris, p. 305-314.

14. Najera, J., 1989. Malaria and the work of WHO, Bulletin of the World Health Organization 67(3), p. 229-243.

15. Nzeyimana I. et al., 2002. Epidémiologie du paludisme dans le sudouest forestier de la Côte d'Ivoire (région de Taï), épidémiologie $\mathrm{n}^{\circ} 2344,94 \mathrm{p}$.

16. OMS, 1996. La situation du paludisme dans le monde en 1993, Weekly Epidemic Rec; 17-22.

17. OMS, 2009. Paludisme dans le monde, Rapport.

18. OMS, 2010. Rapport sur la santé dans le monde.

19. OMS. 2015. Changement climatique et santé. Rapport

20. Patz, J. et al., 2006. Malaria risk and temperature: Influences from global climate change and local land use practices, Proceedings of the National Academy of Sciences of the USA, 103(15), p. 56355636.

21. PNDS, 1996. Programme National de Développement Sanitaire, Rapport.

22. PNLP, 2008. Politique nationale de lutte contre le paludisme, Rapport.

23. PNLP (2010). Manuel de formation à la prise en charge $d u$ paludisme au niveau du District, manuel de l'apprenant, $67 \mathrm{p}$.

24. Rey G. et al., 2007. International Archives of Occupational and Environmental Health, 80 (7): 615-26.

25. Trape J., 2005. Étude de l'impact du changement climatique sur les maladies à transmission vectorielle en Afrique de l'Ouest : le cas de la borréliose à tiques et du paludisme, Rapport final.

26. Valette J-J., 2015. Santé et réchauffement climatique: quelles maladies faut-il redouter in WE DEMAIN. [Http://plus.google.com/share ? www.wedemain.fr/ consulté le 14/03/15

27. Youphil, 2014. Le réchauffement climatique propagerait-il le paludisme [http:/www.youphil.com/fr/taxonomy/term 983] consulté le 04/07/15

28. SIMON P., 2015. Changement climatique : Menaces sur notre santé, in Science et santé, $\mathrm{N}^{\circ} 28$

29. Watts N. et al., 2015. The Lancet, (en ligne) : 10.1016/S01406736(15)60854-6 